Bull. Korean Math. Soc. 50 (2013), No. 4, pp. 1289-1296

http://dx.doi.org/10.4134/BKMS.2013.50.4.1289

\title{
SOME IDENTITIES ON THE BERNSTEIN AND $q$-GENOCCHI POLYNOMIALS
}

\author{
Hyun-MeE Kim
}

\begin{abstract}
Recently, T. Kim has introduced and analysed the $q$-Euler polynomials (see $[3,14,35,37]$ ). By the same motivation, we will consider some interesting properties of the $q$-Genocchi polynomials. Further, we give some formulae on the Bernstein and $q$-Genocchi polynomials by using $p$-adic integral on $\mathbb{Z}_{p}$. From these relationships, we establish some interesting identities.
\end{abstract}

\section{Introduction}

Let $p$ be a fixed odd prime number. Throughout this paper, $\mathbb{Z}_{p}, \mathbb{Q}_{p}$ and $\mathbb{C}_{p}$ will denote the ring of $p$-adic rational integers, the field of $p$-adic rational numbers, and the completion of algebraic closure of $\mathbb{Q}_{p}$, respectively. Let $\mathbb{N}$ be the set of natural numbers and $\mathbb{N}^{*}=\mathbb{N} \cup\{0\}$. The $p$-adic norm is normally defined by $|p|_{p}=1 / p$. As an indeterminate, we assume that $q \in \mathbb{C}_{p}$ with $|1-q|_{p}<1$ (see [1-43]). Let $U D\left(\mathbb{Z}_{p}\right)$ be the space of uniformly differentiable functions on $\mathbb{Z}_{p}$. For $f \in U D\left(\mathbb{Z}_{p}\right)$, the fermionic $p$-adic integral on $\mathbb{Z}_{p}$ is defined by T. Kim as follows:

$$
\begin{aligned}
I_{-1}(f) & =\int_{\mathbb{Z}_{p}} f(x) d \mu_{-1}(x) \\
& =\lim _{n \rightarrow \infty} \sum_{0 \leq x \leq p^{n}-1} f(x) \mu_{-1}\left(x+p^{n} \mathbb{Z}_{p}\right) \\
& =\lim _{n \rightarrow \infty} \frac{1}{p^{n}} \sum_{0 \leq x \leq p^{n}-1} f(x)(-1)^{x}, \quad(\text { see }[1,21,22,25]) .
\end{aligned}
$$

From (1), we can derive the following integral equation on $\mathbb{Z}_{p}$ :

$$
I_{-1}\left(f_{1}\right)=-I_{-1}(f)+2 f(0),
$$

where $f_{1}(x)=f(x+1)($ see $[1,21,22,25])$.

Received September 24, 2012; Revised December 12, 2012

2010 Mathematics Subject Classification. 11S80, 11B68.

Key words and phrases. $q$-Genocchi number, $q$-Genocchi polynomial, Bernstein polynomial, $p$-adic integral. 
As is well known, the Genocchi polynomials are defined by the generating function as follows:

$$
\frac{2 t}{e^{t}+1} e^{x t}=e^{G(x) t}=\sum_{n=0}^{\infty} G_{n}(x) \frac{t^{n}}{n !}
$$

with the usual convention about replacing $G^{n}(x)$ by $G_{n}(x)$. Taking $x=0$ into (3), we get $G_{n}(0)=G_{n}$ is called the $n$-th Genocchi number (see $[1-4,11,12$, $20,24,28,33,34])$. From (3), we have the following recurrence relations of Genocchi numbers as follows:

$$
G_{0}=0 \quad \text { and } \quad(G+1)^{n}+G_{n}=2 \delta_{1, n},
$$

where $\delta_{1, n}$ is the Kronecker symbol and $n \in \mathbb{N}^{*}$ (see $[2,28,36]$ ).

As is well known, the Frobenius-Euler polynomials, $H_{n}(u \mid x)$, are defined by the generating function as follows:

(5) $\frac{1-u}{e^{t}-u} e^{x t}=\sum_{n=0}^{\infty} H_{n}(u \mid x) \frac{t^{n}}{n !}, u \in \mathbb{C}_{p}$ with $u \neq 1 \quad$ (see $[6,16,25,32,39]$ ).

In the special case, $x=0, H_{n}(u \mid 0)=H_{n}(u)$ is called the $n$-th Frobenius-Euler number (see $[6,16,25,32,39]$ ). For $n, k \in \mathbb{N}^{*}$ with $n>k$ and $x \in \mathbb{Z}_{p}$, the Bernstein polynomials of degree $n$ is defined by

(6) $\quad B_{k, n}(x)=\left(\begin{array}{l}n \\ k\end{array}\right) x^{k}(1-x)^{n-k}=\left(\begin{array}{c}n \\ n-k\end{array}\right)(1-x)^{n-k} x^{k}=B_{n-k, n}(1-x)$

(see $[19,32,33,35,37])$.

In this paper, we investigate some identities for the $q$-Genocchi numbers and polynomials by using $p$-adic integral on $\mathbb{Z}_{p}$. From these relationships, we establish some interesting identities in the next section.

\section{Some identities on the Bernstein and $q$-Genocchi polynomials}

In this section, we assume that $q \in \mathbb{C}_{p}$ with $|1-q|_{p}<1$. As is well known, the $q$-Genocchi polynomials are defined by the generating function as follows:

$$
\frac{2 t}{q e^{t}+1} e^{x t}=e^{G_{q}(x) t}=\sum_{n=0}^{\infty} G_{n, q}(x) \frac{t^{n}}{n !},
$$

with the usual convention about replacing $G_{q}^{n}(x)$ by $G_{n, q}(x)$. In the special case, $x=0$, then we have $G_{n, q}(0)=G_{n, q}$ is called the $n$-th $q$-Genocchi number (see $[1,4,11,20,24,33,34])$. From (7), we have the following recurrence relations of $q$-Genocchi numbers as follows:

$$
G_{0, q}=0 \quad \text { and } \quad q\left(G_{q}+1\right)^{n}+G_{n, q}=2 \delta_{1, n} .
$$

From (8), we easily see that

$$
G_{1, q}=\frac{2}{[2]_{q}}, \quad \lim _{q \rightarrow 1} G_{1, q}=G_{1}, \quad \text { and } \quad G_{2, q}=-\frac{2^{2} q}{[2]_{q}^{2}}
$$


SOME IDENTITIES ON THE BERNSTEIN AND q-GENOCCHI POLYNOMIALS 1291 where $[x]_{q}=\frac{1-q^{x}}{1-q}$ and $x \in \mathbb{Z}_{p}$. By the definition of $q$-Genocchi numbers, we note that

$$
G_{n, q}(x)=\sum_{l=0}^{n}\left(\begin{array}{l}
n \\
l
\end{array}\right) G_{l, q} x^{n-l}
$$

From (8), we get

$$
q\left(G_{q}+1\right)^{n}+G_{n, q}=q G_{n, q}(1)+G_{n, q}=2 \delta_{1, n} .
$$

From (10) and (11), we have

$$
\begin{aligned}
q G_{n, q}(2) & =q\left(G_{q}+2\right)^{n}=q\left(G_{q}+1+1\right)^{n} \\
& =q \sum_{l=0}^{n}\left(\begin{array}{l}
n \\
l
\end{array}\right)\left(G_{q}+1\right)^{l}=q \sum_{l=0}^{n}\left(\begin{array}{l}
n \\
l
\end{array}\right) G_{l, q}(1) .
\end{aligned}
$$

By (11) and (12), we can derive the following equation:

$$
\begin{aligned}
q^{2} G_{n, q}(2) & =q^{2}\left(G_{q}+2\right)^{n}=q^{2}\left(G_{q}+1+1\right)^{n} \\
& =q \sum_{l=0}^{n}\left(\begin{array}{l}
n \\
l
\end{array}\right) q\left(G_{q}+1\right)^{l}=q \sum_{l=1}^{n}\left(\begin{array}{l}
n \\
l
\end{array}\right) q G_{l, q}(1) \\
& =q \sum_{l=2}^{n}\left(\begin{array}{l}
n \\
l
\end{array}\right) q G_{l, q}(1)+q\left[\left(\begin{array}{c}
n \\
1
\end{array}\right) q G_{1, q}(1)\right] \\
& =-q \sum_{l=2}^{n}\left(\begin{array}{l}
n \\
l
\end{array}\right) G_{l, q}+n q\left(2-G_{1, q}\right) \\
& =-q \sum_{l=0}^{n}\left(\begin{array}{l}
n \\
l
\end{array}\right) G_{l, q}+2 n q=-q\left(G_{q}+1\right)^{n}+2 n q \\
& =-q G_{n, q}(1)+2 n q=-2 \delta_{1, n}+G_{n, q}+2 n q .
\end{aligned}
$$

From (13), we have the following theorem.

Theorem 1. For $n \in \mathbb{N}^{*}$, we have

$$
q^{2} G_{n, q}(2)=G_{n, q}+2 n q-2 \delta_{1, n} .
$$

Corollary 2. For $n \in \mathbb{N}$ with $n \geq 2$, we have

$$
q^{2} G_{n, q}(2)=G_{n, q}+2 n q .
$$

By (7) and (8), we can derive the following equation:

$$
\frac{2 t}{q e^{t}+1} e^{x t}=\sum_{n=0}^{\infty} G_{n, q}(x) \frac{t^{n}}{n !}=\sum_{n=1}^{\infty} G_{n, q} \frac{t^{n}}{n !}=\sum_{n=0}^{\infty} \frac{G_{n+1, q}}{n+1} \frac{t^{n+1}}{n !} .
$$

Also, we note that

(15) $\frac{2 t}{q e^{t}+1} e^{x t}=\left(\frac{2 t}{1+q}\right)\left(\frac{1+q^{-1}}{e^{t}+q^{-1}}\right) e^{x t}=\frac{2}{[2]_{q}} \sum_{n=0}^{\infty} H_{n}\left(-q^{-1}\right) \frac{t^{n+1}}{n !}$, 
where $H_{n}\left(-q^{-1}\right)$ are the $n$-th Frobenius-Euler number.

Thus, by (14) and (15), we have

$$
\frac{G_{n+1, q}}{n+1}=\frac{2}{[2]_{q}} H_{n}\left(-q^{-1}\right) .
$$

Therefore, by (16), we obtain the following proposition.

Proposition 3. For $n \in \mathbb{N}^{*}$, we have

$$
\frac{G_{n+1, q}}{n+1}=\frac{2}{[2]_{q}} H_{n}\left(-q^{-1}\right)
$$

where $H_{n}\left(-q^{-1}\right)$ are the $n$-th Frobenius-Euler number.

Let us take $f(x)=q^{x} e^{x t}$. Then, by (2), we get

$$
\int_{\mathbb{Z}_{p}} q^{x} e^{x t} d \mu_{-1}(x)=\sum_{n=0}^{\infty} \frac{G_{n+1, q}}{n+1} \frac{t^{n}}{n !} .
$$

From Proposition 3 and (17), we have the following theorem.

Theorem 4. For $n \in \mathbb{N}^{*}$, we have

$$
\int_{\mathbb{Z}_{p}} q^{x} x^{n} d \mu_{-1}(x)=\frac{G_{n+1, q}}{n+1}=\frac{2}{[2]_{q}} H_{n}\left(-q^{-1}\right) .
$$

By (2), (7), and (17), we have

$$
\begin{aligned}
\int_{\mathbb{Z}_{p}} q^{y}(x+y)^{n} d \mu_{-1}(y) & =\sum_{l=0}^{n}\left(\begin{array}{l}
n \\
l
\end{array}\right) x^{n-l} \int_{\mathbb{Z}_{p}} q^{y} y^{l} d \mu_{-1}(y) \\
& =\sum_{l=0}^{n}\left(\begin{array}{c}
n \\
l
\end{array}\right) x^{n-l} \frac{G_{l+1, q}}{l+1} \\
& =\sum_{l=1}^{n+1}\left(\begin{array}{c}
n \\
l-1
\end{array}\right) x^{n+1-l} \frac{G_{l, q}}{l} \\
& =\frac{1}{n+1} \sum_{l=1}^{n+1}\left(\begin{array}{c}
n+1 \\
l
\end{array}\right) x^{n+1-l} G_{l, q} \\
& =\frac{1}{n+1} \sum_{l=0}^{n+1}\left(\begin{array}{c}
n+1 \\
l
\end{array}\right) x^{n+1-l} G_{l, q} \\
& =\frac{1}{n+1} G_{n+1, q}(x) .
\end{aligned}
$$

From (18), we obtain the following theorem.

Theorem 5. For $n \in \mathbb{N}^{*}$, we have

$$
\int_{\mathbb{Z}_{p}} q^{y}(x+y)^{n} d \mu_{-1}(y)=\frac{1}{n+1} G_{n+1, q}(x)=\frac{2}{[2]_{q}} H_{n}\left(-q^{-1} \mid x\right) .
$$


SOME IDENTITIES ON THE BERNSTEIN AND $q$-GENOCCHI POLYNOMIALS 1293

Now, we consider the symmetric property for the $q$-Genocchi polynomials as follows:

$$
\begin{aligned}
q \sum_{n=0}^{\infty} G_{n, q}(1-x) \frac{t^{n}}{n !} & =\frac{2 q t}{q e^{t}+1} e^{(1-x) t} \\
& =-\frac{-2 t}{1+q^{-1} e^{-t}} e^{-x t} \\
& =-\sum_{n=0}^{\infty} G_{n, q^{-1}}(x) \frac{(-t)^{n}}{n !} \\
& =\sum_{n=0}^{\infty} G_{n, q^{-1}}(x)(-1)^{n+1} \frac{t^{n}}{n !} .
\end{aligned}
$$

From (19), we get

$$
q \sum_{n=0}^{\infty} G_{n, q}(1-x) \frac{t^{n}}{n !}=\sum_{n=0}^{\infty} G_{n, q^{-1}}(x)(-1)^{n+1} \frac{t^{n}}{n !} .
$$

Therefore, we have the following theorem.

Theorem 6. For $n \in \mathbb{N}^{*}$, we have

$$
q G_{n, q}(1-x)=(-1)^{n+1} G_{n, q^{-1}}(x) .
$$

For $n \in \mathbb{N}^{*}$ with $n \geq 2$, by Theorems $4,5,6$, and Corollary 2, we have

$$
\begin{aligned}
\int_{\mathbb{Z}_{p}} q^{-x}(1-x)^{n-1} d \mu_{-1}(x) & =(-1)^{n-1} \int_{\mathbb{Z}_{p}} q^{-x}(x-1)^{n-1} d \mu_{-1}(x) \\
& =(-1)^{n-1} \frac{G_{n, q^{-1}}(-1)}{n} \\
& =q \frac{G_{n, q}(2)}{n}=\frac{1}{n q}\left(G_{n, q}+2 n q\right) . \\
& =\frac{1}{n q}\left(G_{n, q}+2 n q\right) \\
& =\frac{1}{q} \frac{G_{n, q}}{n}+2 \\
& =\frac{1}{q} \int_{\mathbb{Z}_{p}} q^{x} x^{n-1} d \mu_{-1}(x)+2 .
\end{aligned}
$$

Therefore, by (20), we have the following theorem.

Theorem 7. For $n \in \mathbb{N}^{*}$ with $n \geq 2$, we have

$$
\int_{\mathbb{Z}_{p}} q^{-x}(1-x)^{n-1} d \mu_{-1}(x)=\frac{1}{q} \int_{\mathbb{Z}_{p}} q^{x} x^{n-1} d \mu_{-1}(x)+2 .
$$


Now, let $n, k \in \mathbb{N}^{*}$ with $n>k$. Then, by (6) and Theorem 5 , we see that

$$
\begin{aligned}
I & =\int_{\mathbb{Z}_{p}} B_{k, n}(x) q^{x} d \mu_{-1}(x) \\
& =\int_{\mathbb{Z}_{p}}\left(\begin{array}{l}
n \\
k
\end{array}\right) x^{k}(1-x)^{n-k} q^{x} d \mu_{-1}(x) \\
& =\left(\begin{array}{l}
n \\
k
\end{array}\right) \sum_{l=0}^{n-k}\left(\begin{array}{c}
n-k \\
l
\end{array}\right)(-1)^{n-k-l} \int_{\mathbb{Z}_{p}} x^{l+k} q^{x} d \mu_{-1}(x) \\
& =\left(\begin{array}{l}
n \\
k
\end{array}\right) \sum_{l=0}^{n-k}\left(\begin{array}{c}
n-k \\
l
\end{array}\right)(-1)^{n-k-l} \frac{G_{l+k+1, q}}{l+k+1} .
\end{aligned}
$$

From the same method, we have

$$
\begin{aligned}
I & =\int_{\mathbb{Z}_{p}} B_{n-k, n}(1-x) q^{x} d \mu_{-1}(x) \\
& =\int_{\mathbb{Z}_{p}}\left(\begin{array}{c}
n \\
n-k
\end{array}\right)(1-x)^{n-k} x^{k} q^{x} d \mu_{-1}(x) \\
& =\left(\begin{array}{c}
n \\
n-k
\end{array}\right) \sum_{l=0}^{k}\left(\begin{array}{c}
k \\
l
\end{array}\right)(-1)^{k-l} \int_{\mathbb{Z}_{p}}(1-x)^{n-l} q^{x} d \mu_{-1}(x) \\
& =\left(\begin{array}{l}
n \\
k
\end{array}\right) \sum_{l=0}^{k}\left(\begin{array}{l}
k \\
l
\end{array}\right)(-1)^{k-l}\left[q \int_{\mathbb{Z}_{p}} q^{-x} x^{n-l} d \mu_{-1}(x)\right] \\
& =\left(\begin{array}{l}
n \\
k
\end{array}\right) \sum_{l=0}^{k}\left(\begin{array}{l}
k \\
l
\end{array}\right)(-1)^{k-l}\left[2+q \frac{G_{n-l+1, q^{-1}}}{n-l+1}\right] .
\end{aligned}
$$

Thus, by (21) and (22), we obtain the following theorem.

Theorem 8. For $n, k \in \mathbb{N}^{*}$ with $n>k$, we have

$$
\sum_{l=0}^{n-k}\left(\begin{array}{c}
n-k \\
l
\end{array}\right)(-1)^{n-k-l} \frac{G_{l+k+1, q}}{l+k+1}=\sum_{l=0}^{k}\left(\begin{array}{l}
k \\
l
\end{array}\right)(-1)^{k-l}\left[2+q \frac{G_{n-l+1, q^{-1}}}{n-l+1}\right] .
$$

\section{References}

[1] S. Araci, D. Erdal, and J. J. Seo, A study on the fermionic p-adic q-integral on $\mathbb{Z}_{p}$ associated with weighted q-Bernstein and q-Genocchi polynomials, Abstr. Appl. Anal. 2011 (2011), Article ID 649248, 10 pages.

[2] A. Bayad and T. Kim, Identities for the Bernoulli, the Euler and the Genocchi numbers and polynomials, Adv. Stud. Contemp. Math. 20 (2010), no. 2, 247-253.

[3] _ Identities involving values of Bernstein, q-Bernoulli and q-Euler polynomials, Russ. J. Math. Phys. 18 (2011), no. 2, 133-143.

[4] I. N. Cangul, V. Kurt, H. Ozden, and Y. Simsek, On the higher-order $w-q-G e n o c c h i$ numbers, Adv. Stud. Contemp. Math. 19 (2009), no. 1, 39-57.

[5] I. N. Cangul, H. Ozden, and Y. Simsek, A new approach to $q$-Genocchi numbers and their interpolation functions, Nonlinear Anal. 71 (2009), no. 12, e793-e799. 
[6] M. Can, M. Cenkci, V. Kurt, and Y. Simsek, Twisted Dedekind type sums associated with Barnes' type multiple Frobenius-Euler l-functions, Adv. Stud. Contemp. Math. 18 (2009), no. 2, 135-160.

[7] L. Carlitz, Some arithmetic properties of generalized Bernoulli numbers, Bull. Amer. Math. Soc. 65 (1959), 68-69.

[8] - Note on the integral of the product of several Bernoulli polynomials, J. London Math. Soc. 34 (1959), 361-363.

[9] R. Dere and Y. Simsek, Applications of umbral algebra to some special polynomials, Adv. Stud. Contemp. Math. 22 (2012), no. 3, 433-438.

[10] Genocchi polynomials associated with the Umbral algebra, Appl. Math. Comput. 218 (2011), no. 3, 756-761.

[11] L.-C. Jang, A study on the distribution of twisted q-Genocchi polynomials, Adv. Stud. Contemp. Math. 18 (2009), no. 2, 181-189.

[12] N. S. Jung and C. S. Ryoo, On the twisted $(h, q)$-Genocchi numbers and polynomials associated with weight $\alpha$, Proc. Jangjeon Math. Soc. 15 (2012), no. 1, 1-9.

[13] T. Kim, On the q-extension of Euler and Genocchi numbers, J. Math. Anal. Appl. 326 (2007), no. 2, 1458-1465.

[14] - On p-adic interpolating function for $q$-Euler numbers and its derivatives, J. Math. Anal. Appl. 339 (2008), no. 1, 598-608.

[15] _ On the analogs of Euler numbers and polynomials associated with p-adic qintegral on $\mathbb{Z}_{p}$ at $q=$, J. Math. Anal. Appl. 331 (2007), no. 2, 779-792.

[16] _ Identities involving Frobenius-Euler polynomials arising from non-linear differential equations, J. Number Theory 132 (2012), no. 12, 2854-2865.

[17] - On a q-analogue of the p-adic log gamma functions and related integrals, J. Number Theory 76 (1999), no. 2, 320-329.

[18] _ Note on the Euler q-zeta functions, J. Number Theory 129 (2009), no. 7, 17981804.

[19] $73-82$.

[20] - On the multiple q-Genocchi and Euler numbers, Russ. J. Math. Phys. 15 (2008), no. $4,481-486$.

[21] Some identities on the q-Euler polynomials of higher order and q-Stirling numbers by the fermionic p-adic integral on $\mathbb{Z}_{p}$, Russ. J. Math. Phys. 16 (2009), 484-491.

[22] _ Symmetry of power sum polynomial and multivariate fermionic $p$-adic invariant integral on $\mathbb{Z}_{p}$, Russ. J. Math. Phys. 16 (2009), no. 1, 93-96.

[23] — , q-Bernoulli numbers and polynomials associated with Gaussian binomial coefficients, Russ. J. Math. Phys. 15 (2008), no. 1, 51-57.

[24] - Note on q-Genocchi numbers and polynomials, Adv. Stud. Contemp. Math. 17 (2008), no. 1, 9-15.

[25] - An identity of the symmetry for the Frobenius - Euler polynomials associated with the fermionic $p$-adic invariant $q$-integrals on $\mathbf{Z}_{p}$, Rocky Mountain J. Math. 41 (2011), no. 1, 239-247.

[26] _ q-Volkenborn integration, Russ. J. Math. Phys. 9 (2002), no. 3, 288-299.

[27] T. Kim, B. Lee, S.-H. Lee, and S.-H. Rim, Identities for the Bernoulli and Euler numbers and ploynomials, Ars Combinatorics 107 (2012), 325-337.

[28] B. Kurt, The multiplication formulae for the Genocchi polynomials, Proc. Jangjeon Math. Soc. 13 (2010), no. 1, 89-96.

[29] H. Ozden and Y. Simsek, A new extension of q-Euler numbers and polynomials related to their interpolation functions, Appl. Math. Lett. 21 (2008), no. 9, 934-939.

[30] - Interpolation function of the $(h, q)$-extension of twisted Euler numbers, Comput. Math. Appl. 56 (2008), no. 4, 898-908. 
[31] H. Ozden, Y. Simsek, and H. M. Srivastava, A unified presentation of the generating functions of the generalized Bernoulli, Euler and Genocchi polynomials, Comput. Math. Appl. 60 (2010), no. 10, 2779-2787.

[32] S.-H. Rim and S. J. Lee, Some identities on the twisted $(h, q)$-Genocchi numbers and polynomials associated with q-Bernstein polynomials, Int. J. Math. Math. Sci. 2011 (2011), Art. ID 482840, 8 pp.

[33] S.-H. Rim, J. H. Jeong, S. J. Lee, J. H. Jin, and E. J. Moon, q-Bernstein polynomials associated with q-Genocchi numbers and polynomials, J. Comput. Anal. Appl. 14 (2012), no. $6,1006-1013$.

[34] S.-H. Rim, S. J. Lee, E. J. Moon, and J. H. Jin, On the q-Genocchi numbers and polynomials associated with q-zeta function, Proc. Jangjeon Math. Soc. 12 (2009), no. $3,261-267$.

[35] C. S. Ryoo, Some relations between twisted q-Euler numbers and Bernstein polynomials, Adv. Stud. Contemp. Math. 21 (2011), no. 2, 217-223.

[36] Calculating zeros of the twisted Genocchi polynomials, Adv. Stud. Contemp. Math. 17 (2008), no. 2, 147-159.

[37] Some identities of the twisted $q$-Euler numbers and polynomials associated with q-Bernstein polynomials, Proc. Jangjeon Math. Soc. 14 (2011), 239-248.

[38] Y. Simsek, O. A. Bayad, and V. Lokesha, q-Bernstein polynomials related to $q$-FrobeniusEuler polynomials, l-functions, and q-Stirling numbers, Math. Methods Appl. Sci. 35 (2012), no. 8, 877-884.

[39] Y. Simsek, O. Yurekli, and V. Kurt, On interpolation functions of the twisted generalized Frobenius-Euler numbers, Adv. Stud. Contemp. Math. 15 (2007), no. 2, 187-194.

[40] Y. Simsek, Generating functions of the twisted Bernoulli numbers and polynomials associated with their interpolation functions, Adv. Stud. Contemp. Math. 16 (2008), 251278.

[41] —, q-Hardy-Berndt type sums associated with q-Genocchi type zeta and q-lfunctions, Nonlinear Anal. 71 (2009), no. 12, e377-e395.

$[42] \longrightarrow,(h, q)$-Bernoulli numbers and polynomials related to twisted $(h, q)$-zeta function and L-function, J. Math. Anal. Appl. 324 (2006), no. 2, 790-804.

[43] _ Theorems on twisted L-function and twisted Bernoulli numbers, Adv. Stud. Contemp. Math. 11 (2005), no. 2, 205-218.

Department of General Education

KOOKMIN UNIVERSITY

SEOUl 136-702, Korea

E-mail address: kagness@kookmin.ac.kr 